\title{
Expression of Erwinia chrysanthemi Pectinase Genes pell, pelL, and pelZ During Infection of Potato Tubers
}

\author{
Sylwia Jafra, ${ }^{1}$ Izabela Figura, ${ }^{1}$ Nicole Hugouvieux-Cotte-Pattat, ${ }^{2}$ and Ewa Lojkowska ${ }^{1}$ \\ ${ }^{1}$ Laboratory of Phytopathology, Department of Biotechnology, Intercollegiate Faculty of Biotechnology, \\ University of Gdansk and Medical University of Gdansk, Kladki 24, 80-822 Gdansk, Poland. '2Laboratoire \\ de Genetique Moleculaire des Microorganismes, UMR-CNRS 5577, INSA, Bat. 406, 20, Av. A. Einstein, \\ 69621 Villeurbanne cedex, France \\ Accepted 14 June 1999.
}

\begin{abstract}
Erwinia chrysanthemi mutants, containing transcriptional fusions of one of the minor pectate lyase genes (pell, pell, pelZ) with the reporter gene encoding $\beta$-glucuronidase activity, were studied for their ability to cause disease symptoms and to synthesize pectinases after inoculation of potato tubers. The strains affected in pelI and pell genes displayed reduced virulence on potato tubers, demonstrating the important role of these isoenzymes in soft rot disease. Inactivation of the pelZ gene slightly influences the ability to macerate. Analysis of the bacterial population showed rapid multiplication of bacteria during infection. Similar kinetics of growth were observed for all mutants and for the wild-type strain. Comparison of the mutants and the wild-type strain showed that the pelI, pelL, and pelZ mutants synthesized reduced levels of Pels. The expression of pelZ is fivefold higher in planta than in bacterial cultures. In contrast, both pelI and pelL are highly (10-fold factor) induced in planta, which is characteristic of the plant-inducible pectate lyases.
\end{abstract}

The pathogenic bacterium Erwinia chrysanthemi causes various diseases in a wide range of plants. Pathogenicity results from the activity of extracellular enzymes responsible for the maceration process, which involves the depolymerization of pectins in the plant cell wall. In vitro, E. chrysanthemi produces a set of pectin-degrading enzymes such as pectin methylesterase, pectin acetyl esterase, pectate lyase, pectin lyase. and polygalacturonase (Collmer and Keen 1986; Barras et al. 1994; Shevchik et al. 1997). These pectinases are secreted into the external medium via a common secretion system, which is encoded by the out operon, essential for pathogenicity.

Pectate lyases seem to play a crucial role in the maceration of plant tissue (Barras et al. 1994). They cleave internal glycosidic bonds in polygalacturonic acid (PGA) or lowmethylesterified pectins by $\beta$-elimination and exhibit reduced activity on a highly methylesterified or acetylesterified substrate. The pectin methylesterases and pectin acetyl esterases remove the esterified groups from pectin, liberating PGA, which is the substrate of pectate lyases (Laurent et al. 1993; Shevchik and Hugouvieux-Cotte-Pattat 1997). In E. chrysan-

Corresponding author: Ewa Lojkowska; Telephone: +48 58320 2248; Fax: +48 58301 2708; E-mail: lojkowska@biotech.univ.gda.pl themi 3937, most of the Pel activity results from the cumulative action of the five major isoenzymes, PelA, PelB, PelC, PelD, and PelE (Bertheau et al. 1984; Ried and Collmer 1988 ), encoded by independent genes organized in two clusters on the bacterial chromosome (pelA, pelD, and pelE; pelB and pelC) (Hugouvieux-Cotte-Pattat et al. 1989). Deletion of the five major pel genes of E. chrysanthemi does not totally eliminate the ability to macerate plant tissues (Ried and Collmer 1988). This reveals the presence of a new set of Pels, which are probably synthesized by the bacteria after interaction with plant tissues (Beaulieu et al. 1993; Kelemu and Collmer 1993). With the use of various approaches, three new pel genes were cloned: pelL, pelI, and pelZ (Lojkowska et al. 1995; Alfano et al. 1995; Pissavin et al. 1996; Shevchik et al. 1997). Because of their low activity in synthetic medium they were first described as secondary or minor Pels.

Pectins and their derivatives induce synthesis of Pel isoenzymes. Transcription of the pel genes is regulated by environmental conditions such as growth phase, nitrogen starvation, anaerobiosis, osmolarity, temperature, and catabolite repression (Hugouvieux-Cotte-Pattat et al. 1992). Low concentrations of iron also favor the expression of pel genes (Sauvage and Expert 1994). Analysis of transcription of the major pel genes during plant infection shows differences in importance of each isoenzyme in pathogenicity (Lojkowska et al. 1993; Dorel et al. 1996; Masclaux et al. 1996). E. chrysanthemi attacks a wide range of plants and the role of each pectinase depends on the host plant (Beaulieu et al. 1993). The synthesis of each Pel in macerated tissues differs in various plant species (Beaulieu et al. 1993) and also in various types of tissues (Lojkowska et al. 1993). The variety of Pel isoenzymes may result from specific adaptations of the bacteria to macerate different plant tissues. Each Pel has particular individual properties (Lojkowska et al. 1995; Shevchik et al. 1997; Tardy et al. 1997; Pissavin et al. 1998) and can therefore be more or less adapted to the substrate and conditions present in the plant during the first step of infection. The minor pectate lyases PelL, PelI, and PelZ, despite their low activity on purified substrates, can play an important role during infection (Lojkowska et al. 1995; Shevchik et al. 1997; Pissavin et al. 1996).

Plant factors may influence the expression of genes involved in pathogenicity (Boccara et al. 1988; Beaulieu et al. 1993; Dorel et al. 1996). In this study, we have analyzed the involvement of the pelL, pelI, and pelZ genes in pathogenicity 
by inoculating potato tubers with the appropriate pelL::uidA, pelI::uidA, or pelZ::uidA mutant. Using these genetic fusions, we have also compared the expression of pelL, pelI, and pelZ genes in rotting tuber tissues with that of the major Pel genes pelA and pelE.

\section{RESULTS}

\section{Pathogenicity of the mutants on potato tubers.}

Potato tubers were inoculated with the E. chrysanthemi wild-type strain 3937, the mutant A1744, which is deleted for six pel genes ( $\Delta$ pel) (Table 1), and mutants containing a uidA transcriptional fusion in pelI, pelL, and pelZ genes. This type of analysis was previously performed for the major pel genes (Lojkowska et al. 1993), and in this study we used pelA and pelE fusions as references.

Table 1. Erwinia chrysanthemi strains and bacteriophage

\begin{tabular}{|c|c|c|}
\hline Strain & Characteristic & Reference or source \\
\hline 3937 & Wild type & Laboratory collection \\
\hline A1888 & pelA::uidA, $\mathrm{Km}^{\mathrm{r}}$ & $\begin{array}{l}\text { Hugouvieux-Cotte-Pattat et al. } \\
1992\end{array}$ \\
\hline A1828 & pelE::uidA, $\mathrm{Km}^{\mathrm{r}}$ & $\begin{array}{l}\text { Hugouvieux-Cotte-Pattat et al. } \\
\quad 1992\end{array}$ \\
\hline A3102 & pell::uidA, $\mathrm{Km}^{\mathrm{r}}$ & This work \\
\hline A3103 & $\mathrm{A} 1744$, pell:: uidA, $\mathrm{Km}^{\mathrm{r}}$ & This work \\
\hline A1990 & pelL::uidA, $\mathrm{Km}^{\mathrm{r}}$ & Lojkowska et al. 1995 \\
\hline A1994 & A1744, pelL::uidA, $\mathrm{Km}^{\mathrm{r}}$ & Lojkowska et al. 1995 \\
\hline A1954 & pelZ::uidA, $\mathrm{Km}^{\mathrm{r}}$ & Pissavin et al. 1996 \\
\hline A1744 & $\begin{array}{l}\text { PMV4116, } \triangle \text { pelADEpaeY, } \\
\quad \triangle \text { pelBCZ }\end{array}$ & Beaulieu et al. 1993 \\
\hline A2701 & lacZ2 pelI::uidA, $\mathrm{Km}^{\mathrm{r}}$ & Shevchik et al. 1997 \\
\hline \multicolumn{3}{|l|}{ Phage } \\
\hline$\Phi E C 2$ & $\begin{array}{l}\text { General transducing phage } \\
\text { of } E \text {. chrysanthemi }\end{array}$ & Resibois et al. 1984 \\
\hline
\end{tabular}

Rotting, estimated by the diameter of decay, progressed linearly (Fig. 1). After $24 \mathrm{~h}$, the mutants affected in the gene of a minor Pel (pelI, pelL, or pelZ) exhibited a low maceration capacity. The disease severity of the pelA and pelE mutants was intermediate between that observed for the other mutants and the wild-type strain. After $48 \mathrm{~h}$, the pelZ mutant exhibited a maceration similar to that of the pelA and pelE mutants, while the pelI and pelL mutants remained weak in macerating ability. After $72 \mathrm{~h}$, about $40 \%$ reduction of maceration was observed after inoculation of potato tubers with the pelI and pelL mutants, compared with the wild-type strain (Fig. 1). No significant difference of ability to macerate was observed with the pelE, pelA, and pelZ mutants (Fig. 1). The $\Delta$ pel mutant A1744 was able to macerate potato tuber tissue with a lower efficiency than single mutants, as maceration was reduced by $75 \%$ after $72 \mathrm{~h}$, compared with the wild-type strain. In strain A1994 ( $\Delta$ pel, pelL) the reduction of maceration caused by pelL inactivation remained visible although this mutant was still able to macerate tuber tissue (Fig. 1). The maceration ability of A1744, which is deleted for the major isoenzymes, together with the clear reduction of maceration caused by either pell or pelL inactivation, indicated the role played by these minor isoenzymes in the soft rot caused by E. chrysanthemi on potato tubers.

To analyze the bacterial multiplication after inoculation, we determined the total number of bacteria per inoculation site. This value is calculated by multiplying the number of bacteria per gram of rotted tissue (obtained by dilution plating) by the weight of rotted tissue.

Analysis of the bacterial population showed rapid multiplication of bacteria during the first $72 \mathrm{~h}$ after infection. The numbers of bacteria for the wild type and the pelL mutant per gram of rotted tissue are shown in Table 2. Similar kinetics of multiplication per gram of rotted tissue were observed for each mutant (pelA, pelE, pelI, pelL, pelZ) and for the wild-

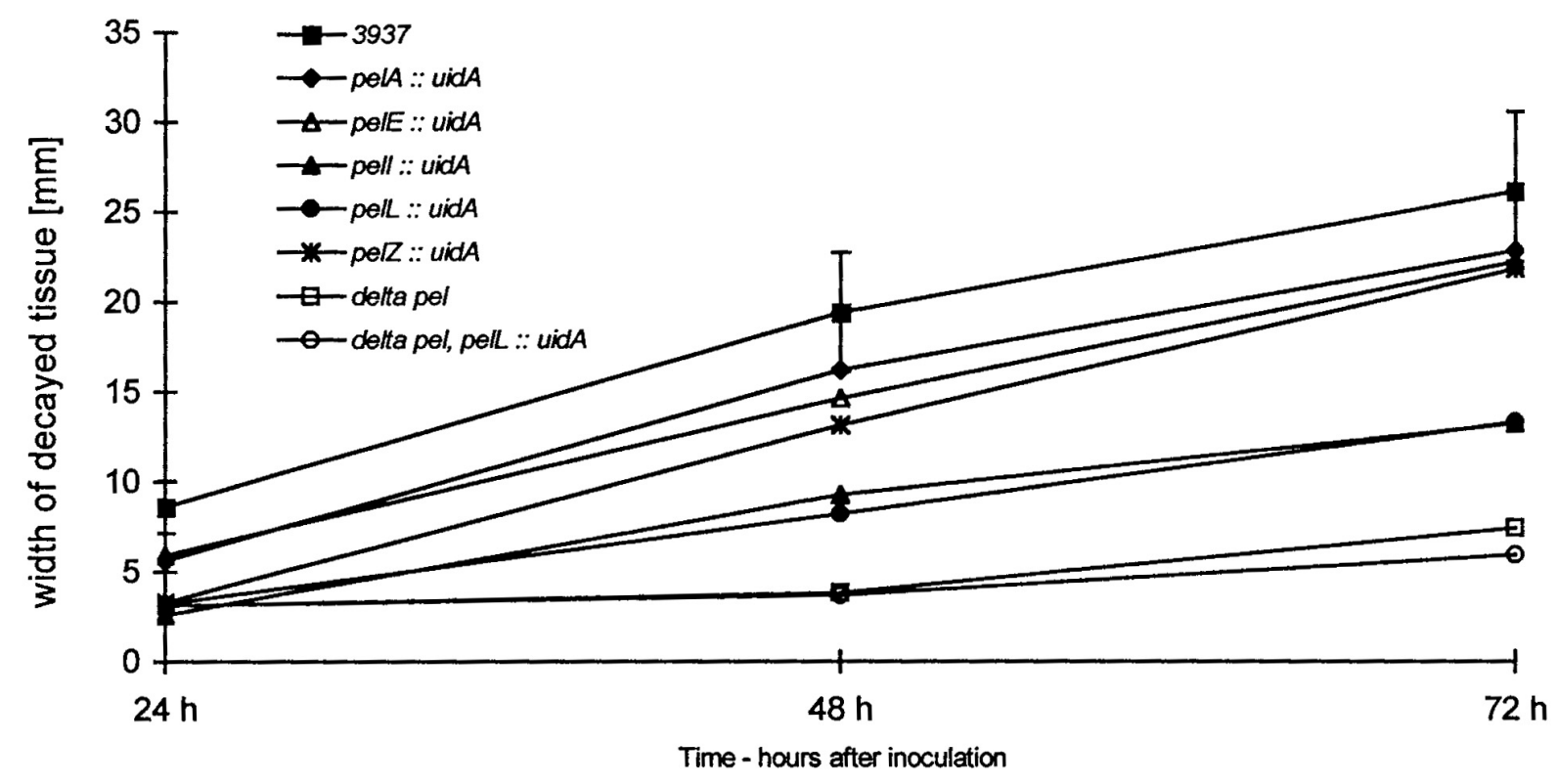

Fig. 1. Comparison of the rotting capacity of the wild-type strain and pel mutants on potato tubers inoculated with the wild-type strain and mutants containing a fusion between one pectinase gene and the reporter gene uidA, using sterile pipette tips containing $50 \mu \mathrm{l}$ of bacterial suspension $\left(2 \times 10^{7}\right.$ $\mathrm{CFU} \cdot \mathrm{ml}^{-1}$ ) and incubated in a dew chamber at $30^{\circ} \mathrm{C}$. Disease severity was estimated by the rot progression. Every $24 \mathrm{~h}$, the width of decayed tissue was measured. Standard deviation was calculated from three independent experiments for each mutant and was similar for the wild-type strain and mutants. For clarity, error bars are included only for the wild-type strain. 
type strain. In contrast, the total number of bacteria for the wild-type strain per inoculation site is significantly higher than that for the pelL mutant. Analysis of the other mutants confirmed that the number of bacteria per inoculation site correlated with the ability to macerate the plant tissue.

\section{Pectate lyase activity during infection.}

In order to compare the ability of the mutants to produce Pels, estimation of pectate lyase activity in tuber tissue was performed 8, 12, 16, 20, 24, 48, and $72 \mathrm{~h}$ after inoculation (Fig. 2). Comparison of the mutants and the wild-type strain showed that the pelI, pelL, and pelZ mutants synthesized reduced levels of all Pels. Statistical analysis indicated that, each time during the first 3 days after inoculation, these mu-

Table 2. Bacterial multiplication between 8 and $72 \mathrm{~h}$ after inoculation of potato tubers $^{\mathrm{a}}$

\begin{tabular}{lccccc}
\hline & $\begin{array}{c}\text { No. of bacteria per gram } \\
\text { of rotted tissue }\left(\times \mathbf{1 0}^{\mathbf{8}}\right)\end{array}$ & & \multicolumn{2}{c}{$\begin{array}{c}\text { No. of bacteria per } \\
\text { inoculation site }\left(\times \mathbf{1 0}^{\mathbf{8}}\right)\end{array}$} \\
\cline { 2 - 3 } \cline { 5 - 6 } $\begin{array}{l}\text { Time after } \\
\text { inoculation }\end{array}$ & $\begin{array}{c}\text { Wild-type } \\
\text { strain }\end{array}$ & $\begin{array}{c}\text { pelL::uidA } \\
\text { mutant }\end{array}$ & & $\begin{array}{c}\text { Wild-type } \\
\text { strain }\end{array}$ & $\begin{array}{c}\text { pelL::uidA } \\
\text { mutant }\end{array}$ \\
\hline $8 \mathrm{~h}$ & 0.10 & 0.08 & & 0.012 & 0.01 \\
$12 \mathrm{~h}$ & 0.18 & 0.16 & & 0.07 & 0.07 \\
$16 \mathrm{~h}$ & 0.63 & 0.69 & & 0.27 & 0.29 \\
$20 \mathrm{~h}$ & 1.40 & 1.21 & & 0.56 & 0.39 \\
$24 \mathrm{~h}$ & 2.02 & 1.60 & & 1.05 & 0.45 \\
$48 \mathrm{~h}$ & 4.40 & 3.70 & & 7.92 & 3.59 \\
$72 \mathrm{~h}$ & 6.80 & 6.10 & 28.60 & 4.51 \\
\hline
\end{tabular}

${ }^{a}$ Potato tubers were inoculated with sterile pipette tips containing $50 \mu \mathrm{l}$ of bacterial suspension $\left(2 \times 10^{7} \mathrm{CFU} \cdot \mathrm{ml}^{-1}\right)$ and incubated in a dew chamber at $30^{\circ} \mathrm{C}$. Number of bacteria was estimated $8,12,16,20,24$ 48 , and $72 \mathrm{~h}$ after inoculation of potato tubers with all tested strains. Number of bacteria in $1 \mathrm{~g}$ of rotted tissue was determined by dilution plating. Number of bacteria per inoculation site was calculated by multiplying number of bacteria per $1 \mathrm{~g}$ by weight of all rotted tissue. tants produced significantly lower levels of Pels per gram of tissue than the wild-type strain. The low Pel activity in plant tissues inoculated with the pelI and pelL mutants is linked to a decrease of maceration (Figs. 1 and 2). However, despite reduced Pel activity, only a transient reduction of maceration was observed with the pelZ mutant (Figs. 1 and 2).

\section{Expression of the pel genes during infections.}

Estimation of pel gene expression in potato tubers was performed by measuring the $\beta$-glucuronidase (GUS) activity due to the reporter gene uidA. It was possible to differentiate various levels of expression of the fusions: pelL and pelZ are weakly expressed (up to $270 \mathrm{U}$ per gram of tissue), but somewhat higher than pelA (Fig. 3A), whereas pell is highly expressed (up to 2,600 $\mathrm{U}$ per gram of tissue) at a level similar to that of pelE (Fig. 3B).

During the first $16 \mathrm{~h}$, expression of all the fusions is very low and there is no significant difference in their levels. When maceration becomes really apparent (which is at about $20 \mathrm{~h}$ ), pelA, pelL, and pelZ remain weakly expressed (about $40 \mathrm{U}$ per gram of tissue) while pelE and pelI are induced (250 U per gram of tissue). Two days after inoculation pelA is still weakly expressed (60 U per gram of tissue), while pelL and pelZ expression has increased further. In most cases, expression of the fusions reaches a plateau at $48 \mathrm{~h}$. In the case of the pelI fusion, the expression still increases at $72 \mathrm{~h}$ (Fig. 3). The final level of expression of the pelE and pelI genes is about 10 times higher than that of pelL or pelZ.

\section{Comparison of pel gene expression in potato tubers and in bacterial cultures.}

The pel gene expression observed in potato tubers was compared with that obtained in bacterial cultures (Fig. 4). Synthetic media without inducer or with PGA or PGA and

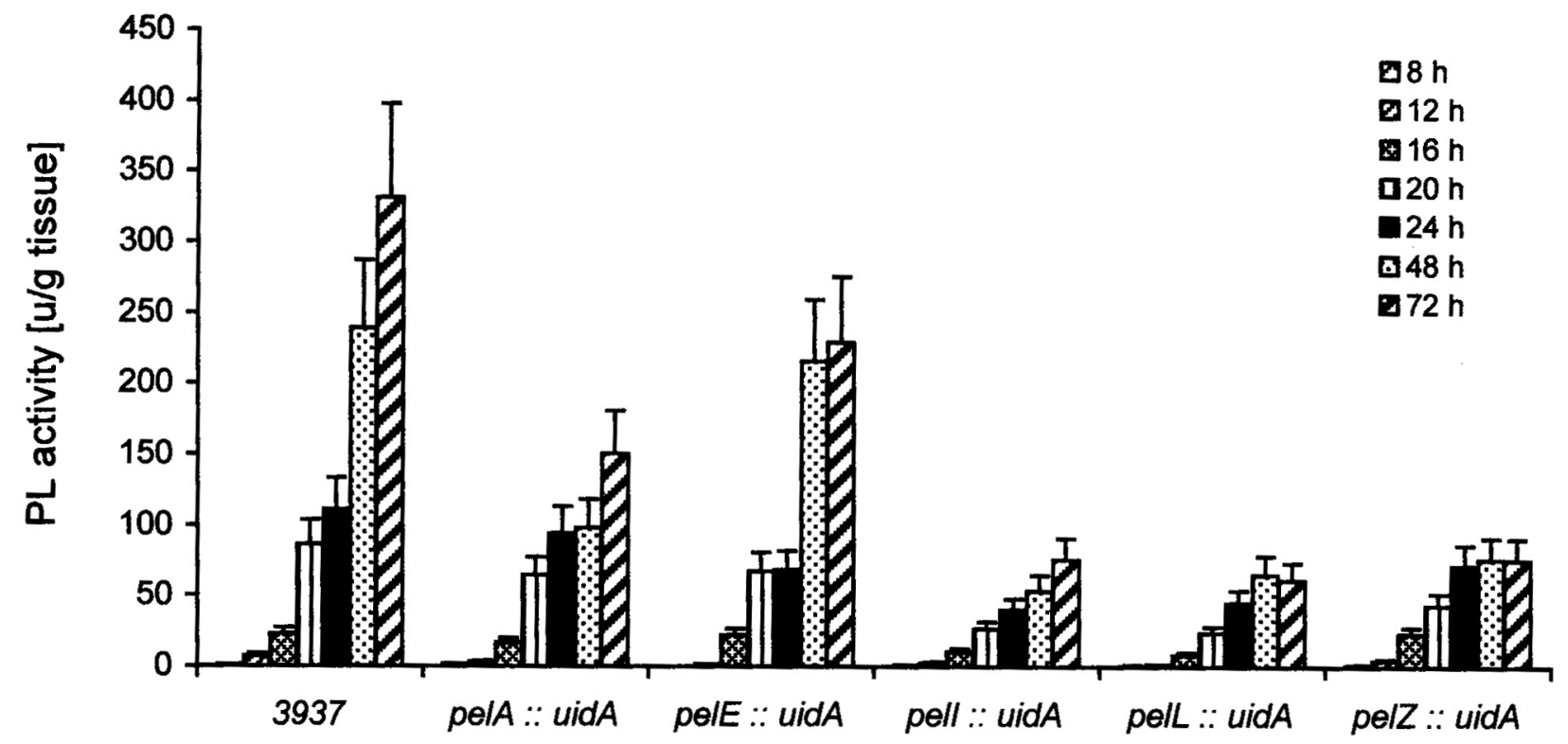

Fig. 2. Activity of pectate lyase after inoculation of potato tubers. The wild-type strain and the three mutants containing a fusion between the minor pel genes and the reporter gene uidA were inoculated into potato tubers as described in Figure 1 caption. Estimation of pectate lyase activity in tuber tissue was performed $8,12,16,20,24,48$, and $72 \mathrm{~h}$ after inoculation in toluenized extracts of infected tissue. Number of bacteria in $1 \mathrm{~g}$ of rotted tissue was determined by dilution plating (three replicates) and the corresponding bacterial dry weight was calculated. Values of Pel activity were expressed per gram of rotting tissue. One unit of Pel activity was defined as the amount of enzyme required for liberation of $1 \mu$ mol of unsaturated product per minute. Error bars indicate the standard deviation calculated from four independent experiments. 
potato tuber extract were inoculated with each strain. With the wild-type strain the global Pel activity was induced in the presence of PGA and presented a higher induction in the presence of both PGA and potato tuber extract (Fig. 4A).

With the pel fusions, the three different levels of expression observed in planta were reproduced in synthetic media: pelI and pelE are expressed more than pelL and pelZ, which in turn are expressed more than pelA. The addition of PGA and PGA with potato tuber extract gave very different effects on the individual pel genes. The pelA and pell fusions were better induced in the presence of PGA and potato tuber extract than with PGA only (Fig. 4B and D). The pelE and pelL fusions showed a better induction in the presence of PGA only (Fig. 4C and E). The pelZ expression was similar in the presence of PGA and of both PGA and potato tuber extract (Fig. 4F). Thus, the pel genes are not all subjected to hyperinduction in the presence of plant extracts.

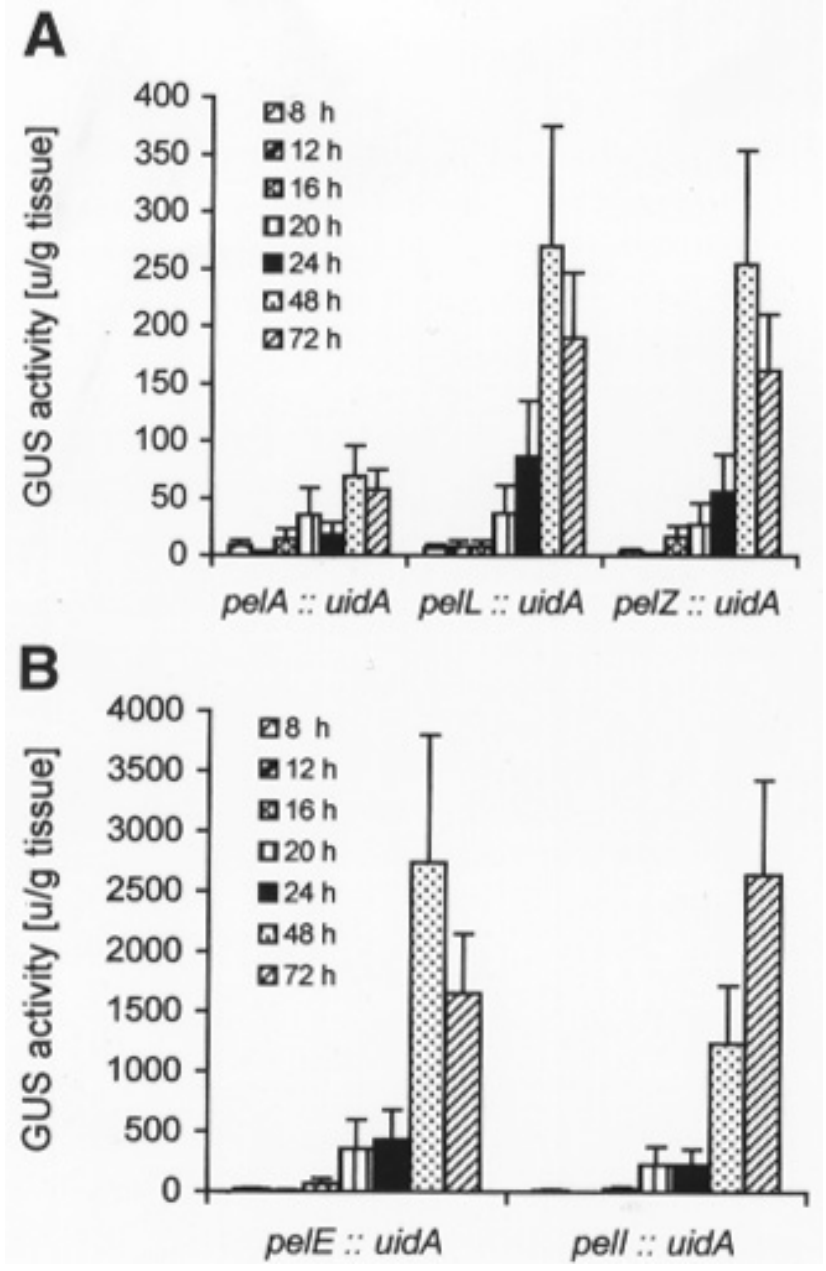

Fig. 3. Expression of the pel::uidA fusions in inoculated tubers tissue. $\beta$ Glucuronidase (GUS) activity was determined $8,12,16,20,24,48$, and $72 \mathrm{~h}$ after inoculation of potato tubers with tested mutants as described in Figure 1 caption. Number of bacteria in $1 \mathrm{~g}$ of rotted tissue was determined by dilution plating (three replicates) and the corresponding bacterial dry weight was calculated. Values of GUS activity were expressed per gram of rotting tissue. GUS-specific activity was expressed as nanomoles of $p$-nitrophenol liberated from $p$-nitrophenyl- $\beta$-Dglucuronide per minute per gram of tissue. A, GUS activity of the weakly expressed fusions pelA::uidA, pelL::uidA, and pelZ::uidA. B, GUS activity of the highly expressed fusions pelE::uidA and pelI::uidA.
Both pectate lyase activity and expression of the fusions were induced more in rotting potato tubers than in synthetic medium with inducer. The in planta global Pel activity is increased by a fivefold factor, compared with PGA medium (Fig. 4A). The in planta induction of the pelA, pelE, and pelZ fusion is also about fivefold higher (Fig. 4B, C, and F), while the difference with the pelI and pelL reached a 10-fold factor (Fig. 4D and E), compared with PGA medium.

\section{DISCUSSION}

Pathogenicity of E. chrysanthemi is linked to the production of plant cell-wall-degrading enzymes. Development of the disease process depends on many environmental conditions (Perombelon and Kelman 1980; Maher and Kelman 1983; Beaulieu et al. 1993). Potato tissue appeared very suitable for investigation of the capacity for maceration of a wide range of E. chrysanthemi mutants (Beaulieu and Van Gijsegem 1992; Lojkowska et al. 1993). In this research, we have compared the importance of three different pectate lyase isoenzymes in maceration of potato tuber tissue.

The ability of E. chrysanthemi 3937 to macerate plant tissue results from the action of at least eight endo-pectate lyases. The disruption of any of the single major pectate lyase genes (pelA, pelB, pelC, pelD, pelE) does not significantly affect the maceration capacity of $E$. chrysanthemi on potato tubers (Fig. 1) (Lojkowska et al. 1993). In addition, an E. chrysanthemi strain in which the five major pel genes have been deleted remained capable of limited maceration on plant tissue (Fig. 1) (Beaulieu et al. 1993; Kelemu and Collmer 1993). This suggested the importance of the minor pectate lyases in the soft rot disease. Preliminary work indicated a role of PelL in pathogenicity since disruption of the corresponding gene decreases the virulence on Saintpaulia (Lojkowska et al. 1995). In contrast, pelI or pelZ mutations did not affect pathogenicity to these plants, but reduced the maceration observed on chicory leaves (Pissavin et al. 1996; Shevchik et al. 1997).

Mutants of E. chrysanthemi with transcriptional fusion of pel genes with the reporter gene uidA were used to investigate the role of the individual minor Pels (PelI, PelL, PelZ) in first steps of bacterial infection on potato tubers. For comparison, pectate lyase activity in the wild-type strain and expression of two major pel genes (pelA and pelE) were also analyzed. The progression of disease severity, bacterial growth, pectate lyase activity (which corresponds with the non-mutated pel genes), and GUS activity (indicating the level of expression of the fused gene) were determined $8,12,16,20,24,48$, and $72 \mathrm{~h}$ after inoculation of potato tubers.

After the first $24 \mathrm{~h}$, all mutants affected in a minor pectate lyase gene (pell::uidA, pelL::uidA, or pelZ::uidA) showed a $50 \%$ decrease of maceration capacity, compared with the wild-type strain (Fig. 1). However, the effect of the pelZ mutation is transient, since, after $72 \mathrm{~h}$, the maceration caused by the pelZ mutant is similar to that caused by the pelE and pelA mutants, whose pathogenicity is not significantly different from that of the wild-type strain (Fig. 1) (Lojkowska et al. 1993). Preliminary results indicated that inactivation of the pelL gene leads to attenuation of the E. chrysanthemi virulence on potato tubers (Lojkowska et al. 1995; Jafra et al. 1997). In our studies, the pelL and pelI mutants showed a significant reduction of the capacity to cause soft rot on potato tubers even $72 \mathrm{~h}$ after infection 
(Fig. 1). These results indicate an important role of both PelL and PelI isoenzymes in E. chrysanthemi pathogenicity.

All the mutants showed active multiplication in planta. Each time after infection, the number of bacteria per gram of

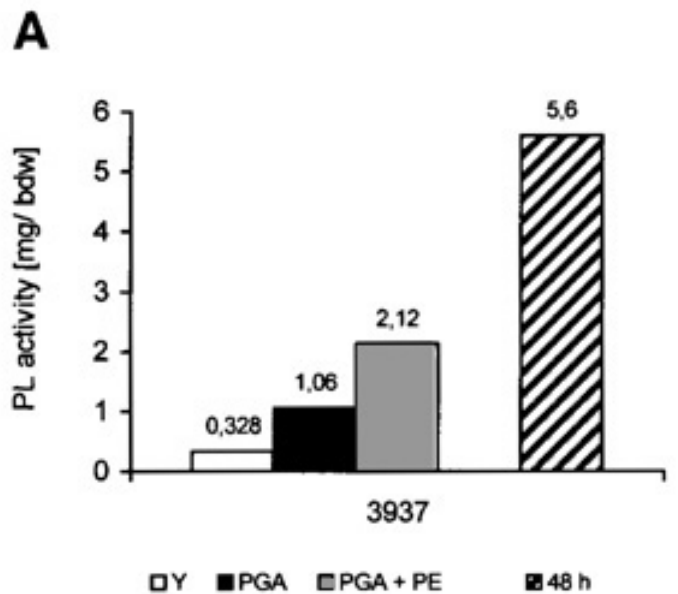

B
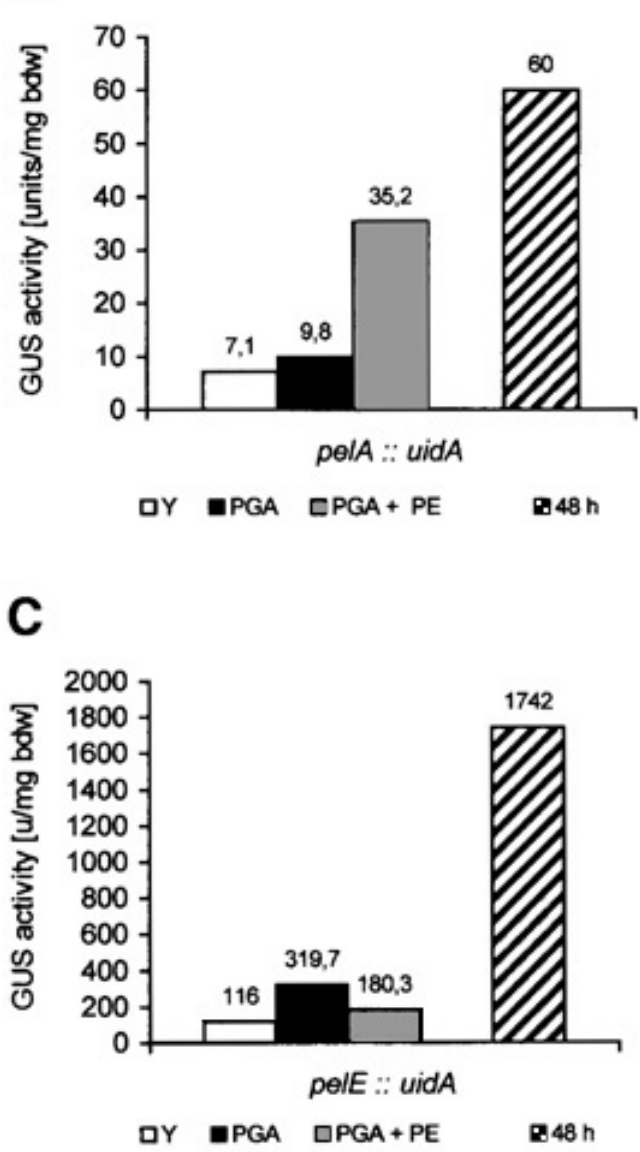

rotted tissue was similar for each strain (Table 2), as observed previously (Lojkowska et al. 1993). In contrast, the number of bacteria per inoculation site of the wild-type strain and the pelL mutant differed and were correlated with disease severity

D

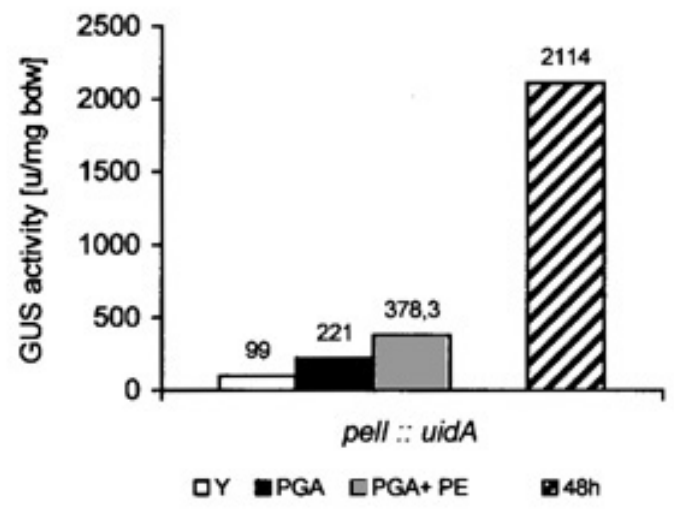

E
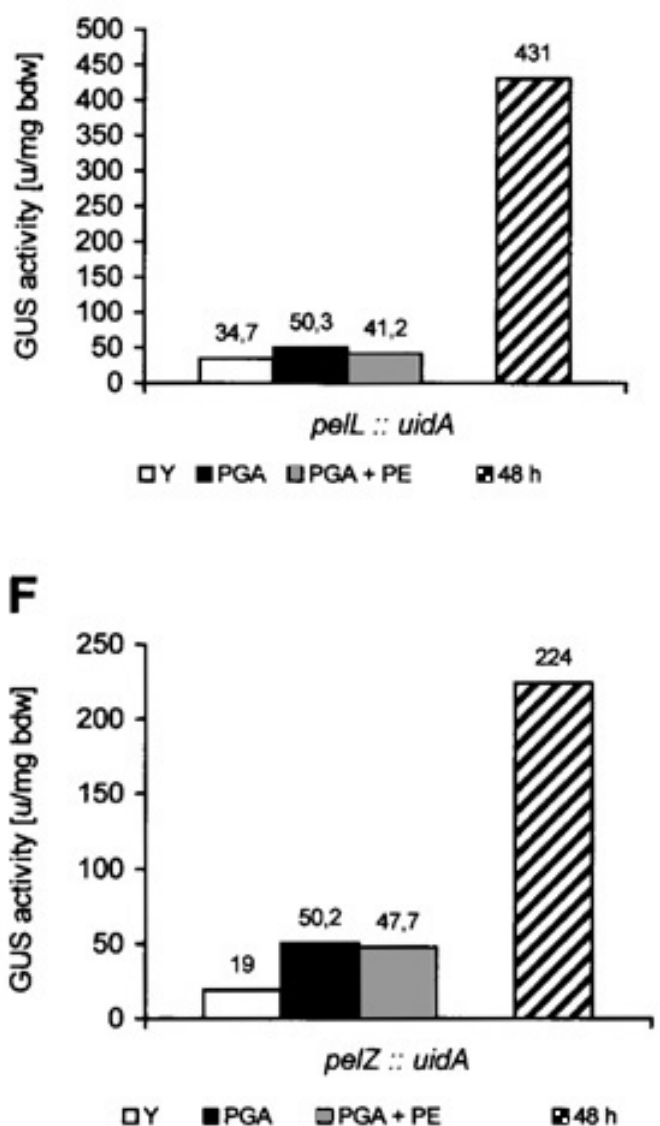

Fig. 4. Expression of pel genes in potato tuber and bacterial cultures. Cultures were grown at $30^{\circ} \mathrm{C}$ in $\mathrm{M} 63+$ glycerol (Y) minimal medium supplemented with $2.5 \mathrm{~g} \cdot$ liter $^{-1}$ polygalacturonic acid (PGA) or $1.5 \%$ potato tubers extract (PE) to late-log phase of growth. The wild-type strain and mutants containing a pel::uidA fusion were inoculated into potato tubers as described in Figure 1 caption. A, Pel activity produced by the wild-type strain in synthetic media and tuber tissue. Pel activity was determined in toluenized extracts of rotted tissue. Specific activity of Pel was expressed as micromoles of unsaturated products liberated per minute per milligram of bacterial dry weight. B-F, $\beta$-glucuronidase (GUS)-specific activity reflected expression of pel genes pelA, pelE, pelI, pelL, pelZ, respectively. GUS activity was determined in toluenized, rotted tissue extracts. Specific GUS activity is expressed as nanomoles of $p$-nitrophenol liberated from $p$-nitrophenyl- $\beta$-D-glucuronide per minute per milligram of bacterial dry weight. 
(Table 2). Such correlation between bacterial multiplication and the ability to macerate tuber tissue was expected.

Disruption of one of the minor pel genes provokes a reduction of 70 to $80 \%$ of the pectate lyase activity in plant tissue (Fig. 2). Damage in one of the major genes influences the total pectate lyase activity to a lower extent, since only a 10 to $60 \%$ reduction was observed (Lojkowska et al. 1993). This indicates that the absence of expression of minor Pels could affect the synthesis of others. Our hypothesis is that minor Pels can attack particular constituents of the pectin matrix present in the plant cell walls, enabling better action of the other pectinases. Indeed, good cleavage of pectin is required for the formation of inducers of pectinolysis (Hugouvieux-Cotte-Pattat et al. 1996) which, in turn, enable use of pectin as a carbon source for bacterial growth. For instance, PelI, which acts at high calcium concentration (Shevchik et al. 1997), could perhaps disrupt calciumbridged pectin chains. Thus, particular properties of minor Pels could explain why inactivation of one minor pel gene results in lower maceration of plant tissues associated with lower induction of Pel synthesis and bacterial multiplication.

Analysis of fusion expression during the infection process enables differentiation of two groups of minor pel genes. Determination of the expression of uidA fusions with pelA, pelE, pelI, pelL, and pelZ indicated that, during the first $16 \mathrm{~h}$ of infection, their induction is very low, but similar. After the first $20 \mathrm{~h}$, the differences become apparent: pelE and pelI are highly expressed; expression of pelL and pelZ is moderate but higher than that of pelA (Fig. 3A and B). The level of pelL and pelZ expression is similar to that of the major pel genes pelB or pelC (Lojkowska et al. 1993).

We have compared the expression of each pel::uidA fusion and total pectate lyase activity of the wild-type strain after inoculation of potato tubers and synthetic medium with or without inducer. Kelemu and Collmer (1993) and Beaulieu et al. (1993) reported that bacteria synthesize the minor Pels only in plant tissue or in the presence of plant extract. In this work, we have observed that for each pel gene the highest induction takes place in plant tissue with increase factors of 10 for pell or pelL and 5 for pelZ, pelA, or pelE (Fig. 4). Comparing with PGA, the presence of plant extract did not increase expression of pelE, pelL, or pelZ, and weakly induced that of pelA or pelI. Thus, the effect of plant extract is very limited, compared with real infection conditions in plant tissue. A compound might be missing from the plant extract or an environmental factor may be different.

Different roles of each Pel in the cell wall degradation process could explain the diversity of the minor E. chrysanthemi Pels. Indeed, PelI, PelL, and PelZ belong to three different families of pectate lyases (families III, IV, and V, respectively) (Lojkowska et al. 1995; Pissavin et al. 1996; Shevchik et al. 1997). In contrast, the five E. chrysanthemi major Pels belong to the large family I of pectate lyases (Henrissat et al. 1995). The low homology observed between PelZ and PelC proteins and the fact that the pelZ gene is adjacent to the pelC gene suggest that PelZ could be a highly divergent member of family I (Pissavin et al. 1998). Indeed, in our study of the pel::uidA fusion on potato tubers, pelZ appeared more related by its behavior to pelA or pelE than to pelI or pelL. The pelZ mutant is weakly affected for maceration, as observed for pelA and pelE mutants (Fig. 1). The expression of pelZ in planta is induced by a fivefold factor, similar to that observed for pelA or pelE induction (Fig. 4). In contrast, both pelI and pelL are induced in planta twice as much as pelA, pelE, and pelZ genes (Fig. 4). These genes indicated the characteristics of plant-inducible pectate lyases described by Kelemu and Collmer (1993). The important finding (from the point of view of disease development) is that the minor pelI and pelL are expressed at high levels in tuber tissue; in the case of pelI, as high as major pelE and much higher than pelA. This suggests a putative role of pelI and pelL in disease development.

\section{MATERIALS AND METHODS}

Bacterial strains, potato tubers, and growth condition.

The derivatives of E. chrysanthemi 3937 used in this work are mutants containing a transcriptional fusion of pelA, pelE, pell, pelL, and pelZ genes with the reporter gene uidA. The strains are listed in Table 1.

The bacteria were grown in LB (Luria-Bertani) medium or M63 synthetic medium (Miller 1972) with glycerol (Y) (2 g · liter $\left.^{-1}\right)$, PGA (2.5 g $\cdot$ liter $\left.^{-1}\right)$ (Sigma Chemical, St. Louis, MO) or potato tuber extract as a carbon source or Pel inducer. Bacteria were grown in liquid medium at a temperature of $30^{\circ} \mathrm{C}$ for $24 \mathrm{~h}$ with shaking. The media were solidified with agar (15 $\mathrm{g} \cdot$ liter $^{-1}$ ) when required. When appropriate, kanamycin was added to the media at a concentration of $20 \mu \mathrm{g} \cdot \mathrm{ml}^{-1}$.

Potato tuber extracts were prepared by homogenization of tubers followed by centrifugation and filtration through membranes. The extract was diluted (1/75) in M63 medium and sterilized by final filtration through a $0.22-\mu \mathrm{m}$ porosity membrane.

Potato tubers (Solanum tuberosum L. 'Mila') were obtained from the experimental fields of the Institute for Potato Research, Bonin, Poland.

\section{Construction of bacterial strains.}

The pelI::uidA fusion from the strain A2701 (Shevchik et al. 1997) was transduced into the wild-type strain 3937 and the $\Delta$ pel mutant A1744 with phage $\Phi E C 2$ (Resibois et al. 1984). Mutants obtained after transduction were selected with kanamycin resistance as a selective marker.

\section{Determination of pathogenicity.}

Potato tubers were washed and immersed twice in $10 \%$ sodium hypochloride (Clorox) for $15 \mathrm{~min}$, rinsed in sterile water, and air dried (Lojkowska et al. 1993). Sterile pipette tips containing $50 \mu \mathrm{l}$ of bacterial suspensions $\left(2 \times 10^{7} \mathrm{CFU} \cdot \mathrm{ml}^{-1}\right)$ were inserted into the tuber parenchyma to a depth of $10 \mathrm{~mm}$ (Maher and Kelman 1983). Depending on the strains and size of the tuber, three or four tips were used for its inoculation. Ten tubers were inoculated with each mutant and incubated in high humidity (approximately $98 \%$ ) at $30^{\circ} \mathrm{C}$. Disease severity was estimated by the progression of rot. Tubers were cut vertically through each inoculation point and the diameter of rotting tissue was measured every $24 \mathrm{~h}$ up to $72 \mathrm{~h}$ following the inoculation.

\section{Enzyme assays.}

Enzyme assays were performed on toluenized extracts of cultures grown up to early stationary phase $(24 \mathrm{~h})$ or in bacterial cells collected from infected potato tuber tissue (this was taken from 10 potato tubers for each strain).

Pectate lyase activity was determined by spectrophotometric monitoring of the ability to form unsaturated products, 
which absorb at $230 \mathrm{~nm}$ (Moran et al. 1968). The assay medium contained PGA $0.05 \%, 0.1 \mathrm{mM} \mathrm{CaCl} 2,100 \mathrm{mM}$ Tris$\mathrm{HCl} \mathrm{pH} \mathrm{8.5.} \mathrm{One} \mathrm{unit} \mathrm{of} \mathrm{Pel} \mathrm{activity} \mathrm{was} \mathrm{defined} \mathrm{as} \mathrm{the}$ amount of enzyme required to produce $1 \mu \mathrm{mol}$ of unsaturated product per minute. Specific Pel activity was expressed as micromoles of unsaturated products liberated per minute per milligram of bacterial dry weight.

GUS activity was measured by the degradation of $p$ nitrophenyl- $\beta$-D-glucuronide (PNPU) into $p$-nitrophenol that absorbs at $405 \mathrm{~nm}$ (Bardonnet and Blanco 1992). Specific GUS activity was expressed as millimoles of $p$-nitrophenol liberated per minute per milligram of bacterial dry weight.

The number of bacteria in $1 \mathrm{~g}$ of rotted tissue was determined by dilution plating and the corresponding bacterial dry weight was calculated. The values of Pel and GUS activity were expressed either per gram of rotting tissue or per inoculation site. In the latter case, the number of bacteria was calculated by multiplying the number of bacteria per $1 \mathrm{~g}$ of rotted tissue and weight of all rotted tissue.

\section{Statistical analysis.}

Mean values and standard errors were calculated from four independent experiments.

\section{ACKNOWLEDGMENTS}

We thank Guy Condemine and Sylvie Reverchon for critical reading of the manuscript. This work was supported by a grant from the Polish State Committee for Scientific Research (KBN) 6P04A 03210, TempusPhare Programme JEP07191-94, and from the Centre National de la Recherche Scientifique and the Ministere de l'Education Nationale, de l'Ensciguement Superieur, de la Recherche et de l'Insertion Professionelle. The ATP Franco-Polonaise 76066 supports the collaboration between N. H.-C.-P. and E. L. The receipt of a fellowship from the French government is gratefully acknowledged by S. J.

\section{LITERATURE CITED}

Alfano, J. R., Ham, H. H., and Collmer, A. 1995. Use of Tn5tac1 to clone a pel gene encoding a highly alkaline, asparagine-rich pectate lyase isoenzyme from an Erwinia chrysanthemi EC16 mutant with deletion affecting the major pectate lyase isoenzymes. J. Bacteriol. 177:4553-4556.

Bardonnet, N., and Blanco, C. 1992. uidA antibiotic resistance cassettes for insertion mutagenesis gene fusion and genetic constructions. FEMS Microbiol. Lett. 93:243-248.

Barras, F., Van Gijsegem, F., and Chatterjee, A. K. 1994. Extracellular enzymes and pathogenesis of soft-rot Erwinia. Annu. Rev. Phytopathol. 32:201-234.

Beaulieu, C., Boccara, M., and Van Gijsegem, F. 1993. Pathogenic behavior of pectinase-defective Erwinia chrysanthemi mutants on different plants. Mol. Plant-Microbe Interact. 6:197-202.

Beaulieu, C., and Van Gijsegem, F. 1992. Pathogenic behavior of several mini-Mu-induced mutants of Erwinia chrysanthemi on different plants. Mol. Plant-Microbe Interact. 5:340-346.

Bertheau, Y., Madgidi-Hervan, E., Kotoujansky, A., Nguyen-The, C., Andro, T., and Coleno, A. 1984. Detection of depolymerase isoenzymes after electrophoresis or electrofocusing, or in titration curves. Anal. Biochem. 139:383-389.

Boccara, M., Diolez, A., Rouve, M., and Kotoujansky, A. 1988. The role of individual pectate-lyase of Erwinia chrysanthemi strain 3937 in pathogenicity on Saintpaulia plants. Physiol. Mol. Plant. Pathol. 33: 95-104.

Collmer, A., and Keen, N. T. 1986. The role of pectic enzymes in plant pathogenesis. Annu. Rev. Phytopathol. 24:383-409.

Dorel, C., Hugouvieux-Cotte-Pattat, N., Robert-Baudouy, J., and Lojkowska, E. 1996. Production of Erwinia chrysanthemi pectinases in potato tubers showing high or low level of resistance to soft rot. Eur. J. Plant Pathol. 102:511-517.
Henrissat, B. S., Heffron, S. E., Yoder, M. D., Lietzke, S. E., and Jurnak, F. 1995. Functional implication of structure-based sequence alignment of proteins in the extracellular pectate lyase superfamily. Plant Physiol. 107:963-976.

Hugouvieux-Cotte-Pattat, N., Condemine, G., Nasser, W., and Reverchon, S. 1996. Regulation of pectinolysis in Erwinia chrysanthemi. Annu. Rev. Microbiol. 50:213-257.

Hugouvieux-Cotte-Pattat, N., Dominguez, H., and Robert-Baudouy, J. 1992. Environmental conditions affect the transcription of the pectinase genes of Erwinia chrysanthemi 3937. J. Bacteriol. 174: 7807-7818.

Hugouvieux-Cotte-Pattat, N., Reverchon, S., and Robert-Baudouy, J. 1989 Expanded linkage map of Erwinia chrysanthemi strain 3937. Mol. Microbiol. 3:573-581.

Jafra, S., Figura, I., Hugouvieux-Cotte-Pattat, N., and Lojkowska, E. 1997. Characterization and role in the pathogenesis of potatoes of a novel pectate lyase from Erwinia chrysanthemi 3937. Pages 511-514 in: Developments in Plant Pathology: Diagnosis and Identification of Plant Pathogens. Vol. 11. E. Dehne, ed. Kluwer Academic Pub., Dordrecht, The Netherlands.

Kelemu, S., and Collmer, A. 1993. Erwinia chrysanthemi EC16 produces a second set of plant-inducible pectate lyase isozymes. Appl. Environ. Microbiol. 59:1756-1761.

Laurent, F., Kotoujansky, A., Labesse, G., and Bertheau, Y. 1993. Characterization and overexpression of the pem gene encoding pectin methylesterase of Erwinia chrysanthemi strain 3937. Gene 131:17-25.

Lojkowska, E., Dorel, C., Reignault, P., Hugouvieux-Cotte-Pattat, N., and Robert-Baudouy, J. 1993. Use of GUS fusion to study the expression of Erwinia chrysanthemi pectinase genes during infection of potato tubers. Mol. Plant-Microbe Interact. 6:488-494.

Lojkowska, E., Masclaux, C., Boccara, M., Robert-Baudouy, J., and Hugouvieux-Cotte-Pattat, N. 1995. Characterization of the gene encoding a secondary pectate lyase of Erwinia chrysanthemi 3937. Mol. Microbiol. 16:1183-1195.

Maher, E. A., and Kelman, A. 1983. Oxygen status of potato tuber tissue in relation to maceration by pectic enzymes of Erwinia carotovora. Phytopathology 73:536-539.

Masclaux, C., Hugouvieux-Cotte-Pattat, N., and Expert, D. 1996. Iron is a triggering factor for differential expression of Erwinia chrysanthemi strain 3937 pectate lyases in pathogenesis of African violets. Mol. Plant-Microbe Interact. 9:198-205.

Miller J. H. 1972. Experiments in Molecular Genetics. Cold Spring Harbor Laboratory, Cold Spring Harbor, NY.

Moran, F., Nasuno, S., and Starr, M. P. 1968. Extracellular and intracellular polygalacturonic acids trans-eliminases of Erwinia carotovora. Arch. Biochem. Biophys. 123:293-306.

Perombelon, M. C. M., and Kelman, A. 1980. Ecology of the soft rot Erwinia. Annu. Rev. Phytopathol. 18:361-367.

Pissavin, C., Robert-Baudouy, J., and Hugouvieux-Cotte-Pattat, N. 1996. Regulation of pelZ, a gene of the pelB-pelC cluster encoding a new pectate lyase of Erwinia chrysanthemi 3937. J. Bacteriol. 178: 7187-7196.

Pissavin, C., Robert-Baudouy J., and Hugouvieux-Cotte-Pattat, N. 1998. Biochemical characterization of PelZ of Erwinia chrysanthemi 3937. Biochem. Biophys. Acta 1383:188-196

Resibois, A., Colet, M., Faelen, M., Schoonejans, E., and Toussaint, A. 1984. Phi-EC2 a new generalized transducing phage of Erwinia chrysanthemi. Virology 137:102-112.

Ried, J. L., and Collmer, A. 1988. Construction and characterization of an Erwinia chrysanthemi mutant with directed deletions in all of the pectate lyase structural genes. Mol. Plant-Microbe Interact. 1:32-38.

Sauvage, C., and Expert, D. 1994. Differential regulation by iron of Erwinia chrysanthemi pectate lyases: Pathogenicity of iron transport regulatory $(c b r)$ mutants. Mol. Plant-Microbe Interact. 7:71-77.

Shevchik, V. E., and Hugouvieux-Cotte-Pattat, N. 1997. Identification of bacterial pectin acetyl esterase in Erwinia chrysanthemi 3937. Mol. Microbiol. 24:1285-1301.

Shevchik, V. E., Robert-Baudouy, J., and Hugouvieux-Cotte-Pattat, N. 1997. Pectate lyase pelI of Erwinia chrysanthemi 3937 belongs to a new family. J. Bacteriol.179:7321-7330.

Tardy, F., Nasser, W., Robert-Baudouy, J., and Hugouvieux-Cotte-Pattat, N. 1997. Comparative analysis of the five major Erwinia chrysanthemi pectate lyases: Enzyme characteristic and potential inhibitors. J. Bacteriol. 179:2503-2511. 